\title{
An unexpected and useful E-ring oxidative cleavage in furostanes
}

\author{
Anielka Rosado-Abon, Margarita Romero-Avila, and Martin A. Iglesias-Arteaga* \\ Departamento de Química Orgánica, Facultad de Química. Universidad Nacional Autónoma de \\ México. Ciudad Universitaria, 04510 México D.F., México \\ E-mail: martin.iglesias@servidor.unam.mx
}

\begin{abstract}
Treatment of a acetylated furostanols derived from steroid sapogenins with a wet mixture of $\mathrm{KMnO}_{4}$ and $\mathrm{Fe}_{2}\left(\mathrm{SO}_{4}\right)_{3} \cdot \mathrm{nH}_{2} \mathrm{O}$ in $\mathrm{CH}_{2} \mathrm{Cl}_{2}$ /tert-butyl alcohol produces the unexpected E-ring oxidative cleavage to afford the corresponding cholestan-22,16-diones. Based in this new reaction, facile syntheses of an OSW-1 precursor and kryptogenin acetate have been designed.
\end{abstract}

Keywords: Oxidative cleavage, furostanes, kryptogenin, OWS-1, 16,22-dioxosteroids

\section{Introduction}

The stereoselective $\beta$-epoxidation of $\Delta^{5}$-steroids using biphasic systems that involve potassium permanganate and metal salts has received considerable attention. ${ }^{1}$ In particular, we have reported the transformation of $3 \beta$-hydroxy- $\Delta^{5}$-steroids into the corresponding $3 \beta$-hydroxy-6-oxosteroids [i.e cholesterol acetate into $3 \beta$-hydroxy-cholestan-6-one and diosgenin acetate into laxogenin], using a protocol that involves $\beta$-epoxidation, $\mathrm{HBr}$ induced oxirane ring opening, oxidation the resulting bromohydrin to a bromoketone and reduction to the parent ketone by treatment with $\mathrm{Zn}$ dust in refluxing acetic acid, (see Scheme 1). ${ }^{2}$ 
<smiles>CC(=O)OC1CCC2(C)C(=CCC(C)C2C)C1</smiles><smiles>CCCCCC1C(C)CC(=O)[C@@H]2CC(O)CCC12C</smiles><smiles>CC(=O)OC1CCC2(C)C(C)C(C)CC(=O)[C@@]2(C)C1</smiles><smiles>CC(C)CC1CCC2(C)C(C)C(C)CC(=O)[C@@]2(Br)C1</smiles>

i) Wet $\mathrm{KMnO}_{4}, \mathrm{Fe}_{2}\left(\mathrm{SO}_{4}\right)_{3}$, tert-BuOH, ii) $\mathrm{CH}_{2} \mathrm{Cl}_{2} / \mathrm{HBr} / \mathrm{CH}_{2} \mathrm{Cl}_{2} /$ iii) Jones oxidation, iv) $\mathrm{Zn} / \mathrm{AcOH}$ reflux, v) $\mathrm{KOH} /$ metanol

\section{Scheme 1}

Attempts to apply the above described procedure to furostanic compounds led to an unexpected oxidative cleavage reaction in which the furostanic compounds were converted into the corresponding cholestan-16,22-diones. Herein we report on the oxidative cleavage of the Ering of furostanes and some of its synthetic applications.

\section{Results and Discussion}

Attempt at $\beta$-epoxidation of the acetylated furostanol 1 a using wet $\mathrm{KMnO}_{4} / \mathrm{Fe}_{2}\left(\mathrm{SO}_{4}\right)_{3}$ in $\mathrm{CH}_{2} \mathrm{Cl}_{2}$ /tert-butyl alcohol afforded the unchanged starting material and two compounds that after separation were characterized as the desired epoxidated furostanol $\mathbf{2 a}(16.6 \%)$ and the unexpected epoxidated diketone 3a (43.3\%), (see Scheme 2 and Table 1, entry 1). In an experiment conducted without the ferric salt the unchanged starting material was recovered after 24 hours.

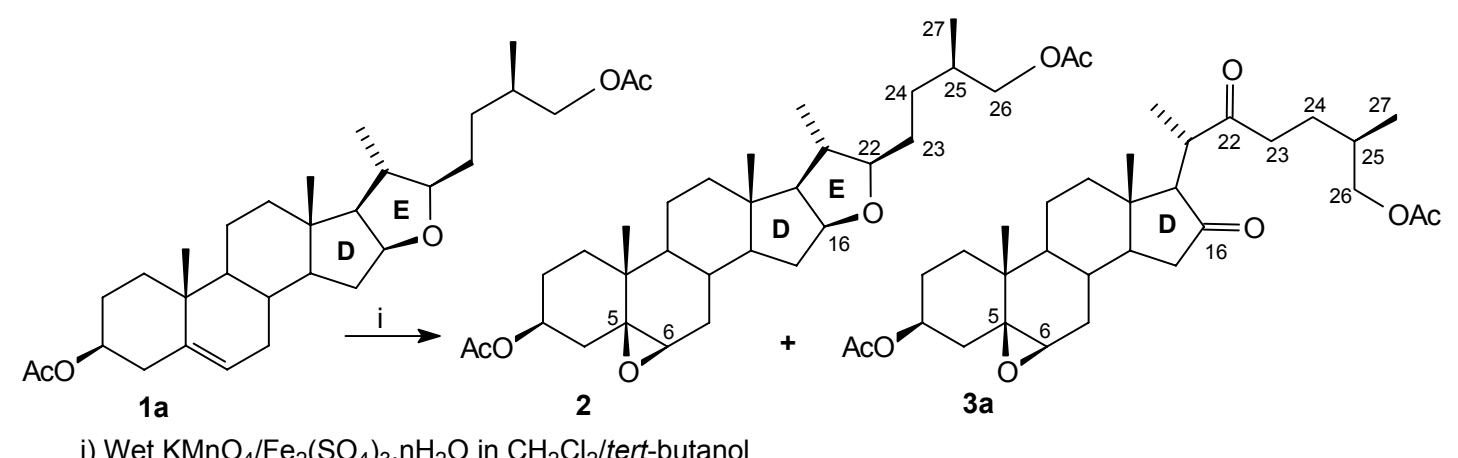

i) Wet $\mathrm{KMnO}_{4} / \mathrm{Fe}_{2}\left(\mathrm{SO}_{4}\right)_{3} \cdot \mathrm{nH}_{2} \mathrm{O}$ in $\mathrm{CH}_{2} \mathrm{Cl}_{2} /$ tert-butanol

\section{Scheme 2}


Some reports have shown that the E-ring of furostanes undergoes cleavage in the presence of Lewis acids. For instance, $\mathrm{Ac}_{2} \mathrm{O} / \mathrm{BF}_{3}$. $\mathrm{Et}_{2} \mathrm{O}$ treatment of furostanes has been reported to produce E-ring cleavage followed by $17 \rightarrow 16$ hydride shift and Wagner-Meerwein rearrangement to afford $\Delta^{13}-17 \beta$-methyl-18-norsteroids (Scheme 3). ${ }^{3}$ Activation of acetic anhydride by $\mathrm{BF}_{3} . \mathrm{Et}_{2} \mathrm{O}$ resulted in acetylation of $\mathrm{O}-16$ that triggers the observed rearrangement. The absence of a nucleophile capable to produce the nucleophilic displacement of the acetylated oxygen, justifies the observed course.

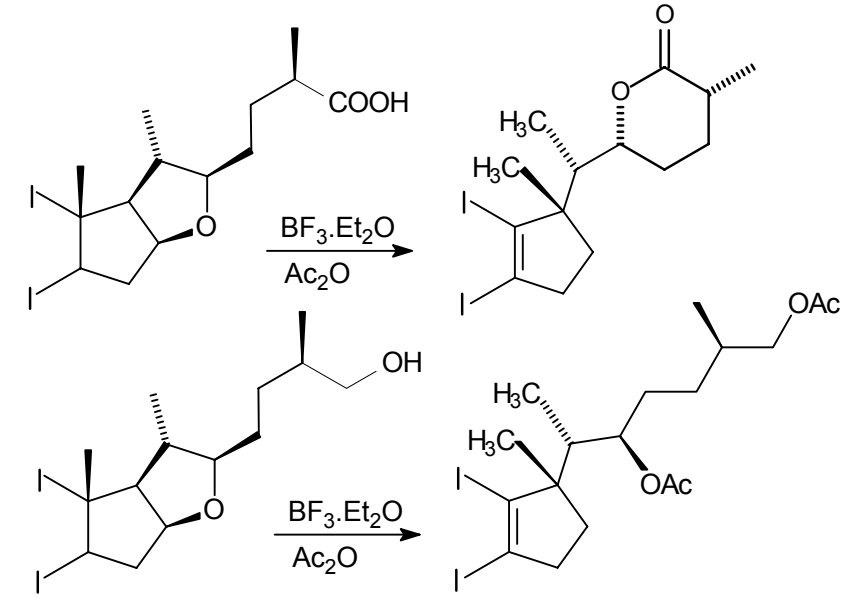

\section{Scheme 3}

In this new reaction of furostanic compounds with wet $\mathrm{KMnO}_{4} / \mathrm{Fe}_{2}\left(\mathrm{SO}_{4}\right)_{3}$, the fact that in absence of the ferric salt no reaction was observed suggests a mechanism in which, after coordination to the ferric cation, the tetrahydrofuranic oxygen attached to both $\mathrm{C}-16$ and C-22 can be displaced by water present in the reaction media leading to a diol which is rapidly oxidized to the observed 16,22-diketone, (Scheme 4).

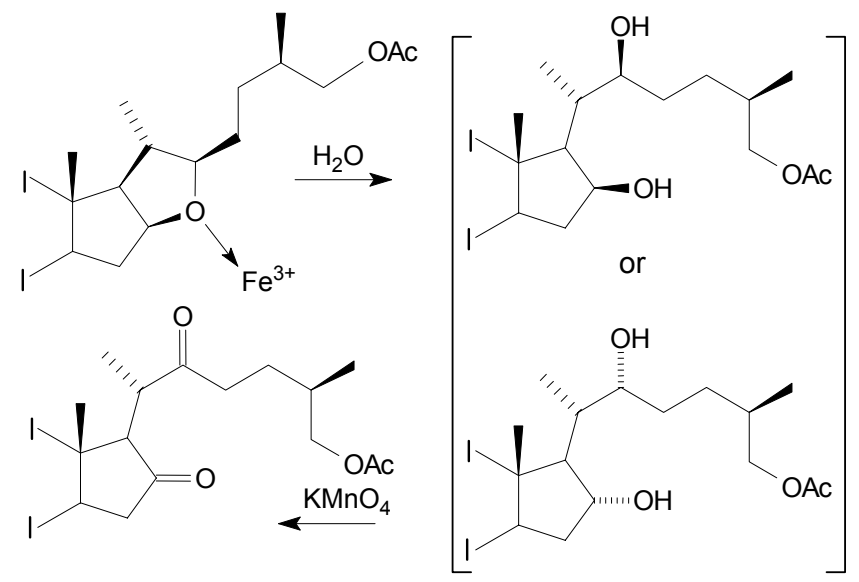

\section{Scheme 4}


The distribution of products indicates that the epoxidation of the double bond is faster than the E-ring cleavage and prompted us to study the reaction conditions for the total conversion of 1a into 3a. We were pleased to find that the simple increase on the amounts of the reagents and reaction time resulted in the conversion of the starting material $\mathbf{1 a}$ into $\mathbf{3 a}$, (see Table 1, entry 2).

Table 1. Results of of epoxidation-oxidative E-ring opening of compound 1a

\begin{tabular}{ccccccc}
\hline & $\mathrm{KMnO}_{4}(\mathrm{~g})$ & $\mathrm{Fe}_{2}\left(\mathrm{SO}_{4}\right)_{3} \cdot \mathrm{nH}_{2} \mathrm{O}(\mathrm{g})$ & $\mathrm{H}_{2} \mathrm{O}(\mathrm{ml})$ & $\mathrm{Time}(\mathrm{min})$. & \multicolumn{2}{c}{$\mathrm{Yield} \%$} \\
& & & & & 2 & $3 \mathrm{a}$ \\
\hline 1 & 4 & 2 & 0.4 & 20 & 16.6 & 49.3 \\
2 & 5 & 2.5 & 0.5 & 120 & - & 84.4 \\
\hline
\end{tabular}

It has been reported that treatment of $\mathbf{1 b}$ with $\mathrm{K}_{2} \mathrm{Cr}_{2} \mathrm{O}_{7}$ in acetic acid led to the 16,22diketone $\mathbf{4 b}$ in poor yields. ${ }^{4 a}$ In an alternative approach, oxidation with Oxone ${ }^{\circledR}$ of the acetylated $i$-steroid 5, derived from diosgenin, produced the hemiketal $\mathbf{6}$ and the desired 16,22-diketone 7. Four times recirculation of the mixture through the same oxidation procedure afforded 7 in $80 \%$ that was isomerised to $\mathbf{4 b}$ in a long sequence to the synthesis of the potent antitumor agent OSW$1 .{ }^{4 \mathrm{~b}}$
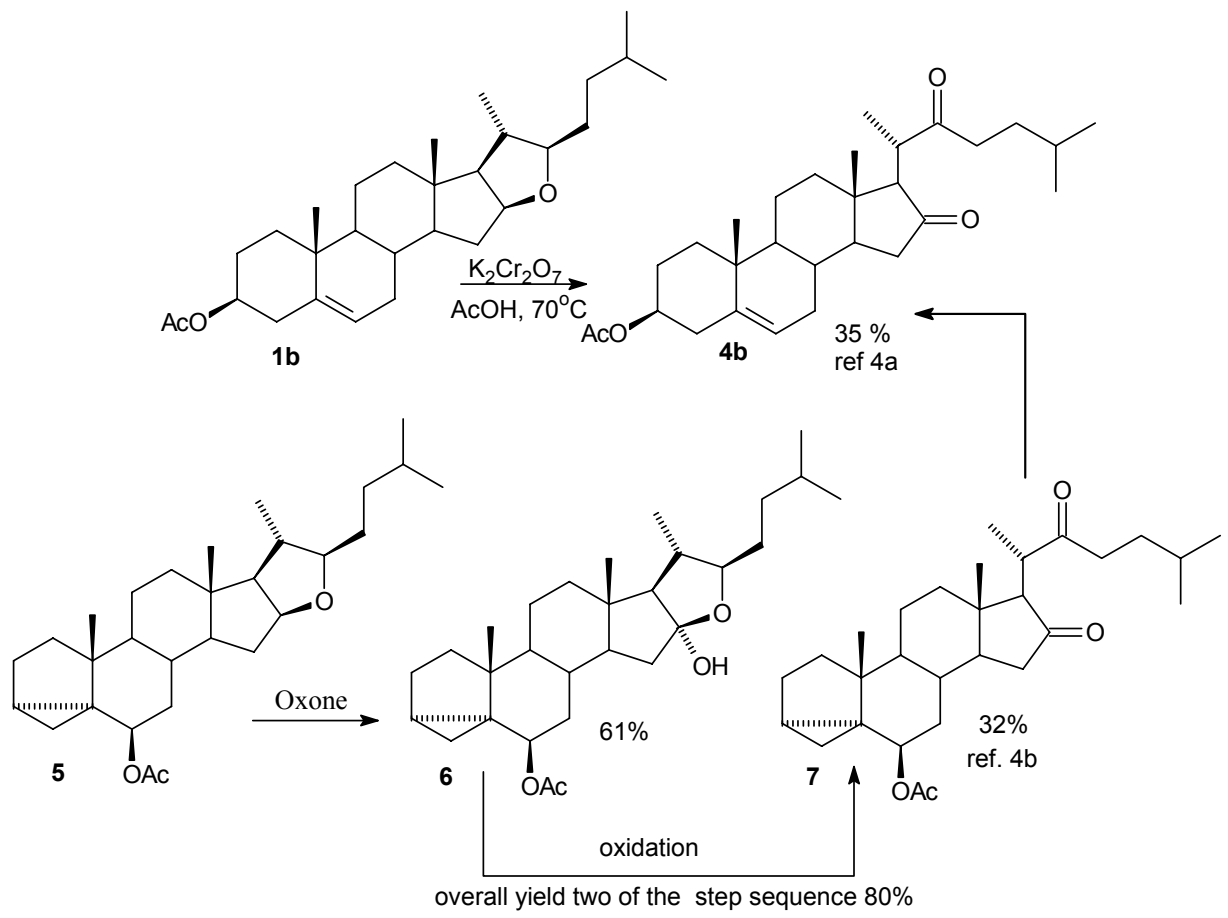

\section{Scheme 5}

After those facts we decided to explore the application of this new reaction to the synthesis of kriptogenin acetate (4a), ${ }^{5}$ and $\mathbf{4 b}$ that has been employed as synthetic precursor of OSW-1. ${ }^{4 b}$ Application of the new reaction conditions to $\mathbf{1 a}$ or to our previously reported $\mathbf{1} \mathbf{b}^{6 \mathrm{~d}}$ led to the 
corresponding epoxidated diketones $\mathbf{3 a}$ and $\mathbf{3 b}$ which were converted into the desired 5,6 unsaturated derivatives $\mathbf{4 a}$ (kryptogenin acetate) and $\mathbf{4 b}$ by consecutive treatments with $\mathrm{HBr}$ in $\mathrm{CH}_{2} \mathrm{Cl}_{2}$ and $\mathrm{Zn}$ dust in refluxing acetic acid.

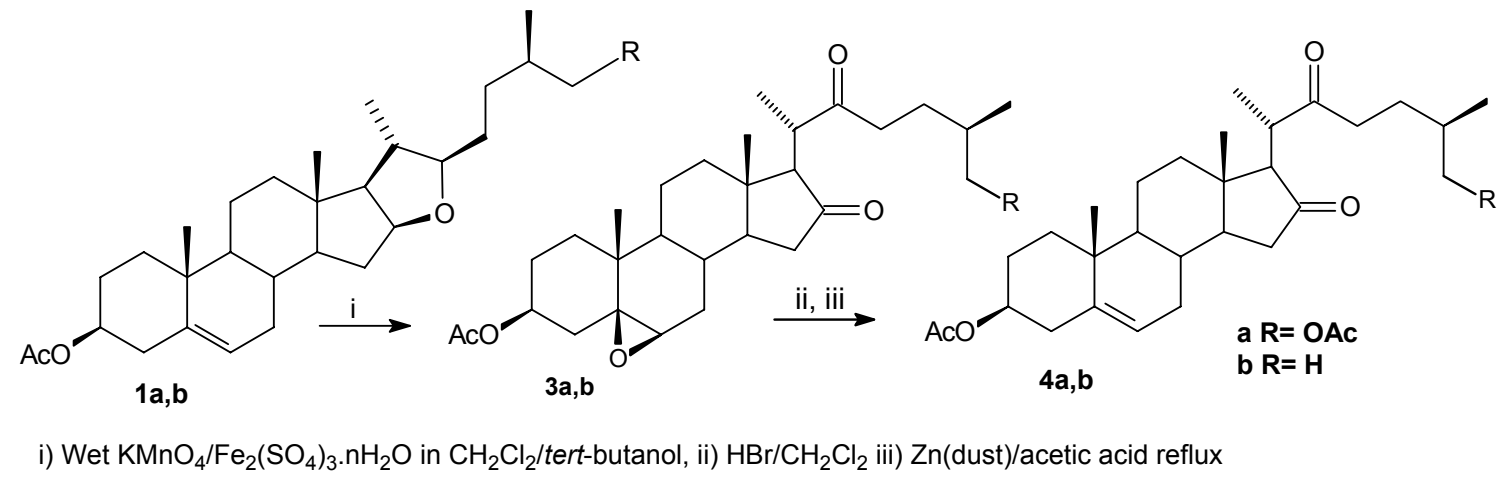

\section{Scheme 6}

\section{Conclusions}

We have found that $\mathrm{KMnO}_{4} / \mathrm{Fe}_{2}\left(\mathrm{SO}_{4}\right)_{3} \cdot \mathrm{nH}_{2} \mathrm{O}$ produces the oxidative cleavage of the E-ring of furostanes to afford a 16,22-diketone. This new reaction constitutes a convenient alternative for the preparation of 16,22-dioxo steroids that may find diverse and interesting synthetic applications. (i.e synthesis of OSW-1, kriptogenin derivatives or novel heterocyclic steroids). Additional experiments to extend this reaction to other steroidal and non-steroidal tetrahydrofuranic compounds are in development.

\section{Experimental Section}

General Procedures. Reactions were monitored by TLC on ALUGRAM ${ }^{\circledR}$ SIL G/UV $2545 \mathrm{~cm} \mathrm{x}$ $2.5 \mathrm{~cm}$ plates from MACHEREY-NAGEL. Chromatographic plates were sprayed with a $1 \%$ solution of vainillin in $50 \% \mathrm{HClO}_{4}$ and heated until colour developed. NMR spectra were recorded in on Varian Unity INOVA (300 or $400 \mathrm{MHz}$ ) spectrometers using TMS for ${ }^{1} \mathrm{H}$ or the solvent signal $\left(\mathrm{CDCl}_{3}\right)$ for ${ }^{13} \mathrm{C}$ as reference. Mass spectra were recorded on a Jeol SX-102-A spectrometer. Melting points were measured on Melt-Temp II equipment and are uncorrected.

\section{Procedure for epoxidation-E-ring cleavage}

$\mathrm{KMnO}_{4}$ and $\mathrm{Fe}_{2}\left(\mathrm{SO}_{4}\right)_{3} \cdot \mathrm{nH}_{2} \mathrm{O}$ were finely grounded in a mortar, $\mathrm{H}_{2} \mathrm{O}$ was added and the mixture was placed in a round bottom flask containing $\mathrm{CH}_{2} \mathrm{Cl}_{2}(10 \mathrm{ml}$ ) (see Table 1 for amounts of $\mathrm{KMnO}_{4}, \mathrm{Fe}_{2}\left(\mathrm{SO}_{4}\right)_{3} \cdot \mathrm{nH}_{2} \mathrm{O}$ and $\left.\mathrm{H}_{2} \mathrm{O}\right)$. A solution of the corresponding furostanic compound ${ }^{6} \mathbf{3 a}$ or 3b $(2 \mathrm{mmol})$ in $\mathrm{CH}_{2} \mathrm{Cl}_{2}(5 \mathrm{ml})$ was added followed by addition of tert-butyl alcohol $(1 \mathrm{ml})$ and 
the mixture was stirred at room temperature for the indicated time. Ethyl ether $(25 \mathrm{ml})$ and celite $(2 \mathrm{~g})$ were added and the mixture was stirred for $15 \mathrm{~min}$ before filtering through a small pad of silicagel and elution with ethyl ether $(25 \mathrm{ml})$ and ethyl acetate $(2 \times 30 \mathrm{ml})$. The filtrate was washed with $\mathrm{H}_{2} \mathrm{O}(5 \times 30 \mathrm{ml})$, saturated aqueous $\mathrm{NaCl}$ solution $(2 \times 30 \mathrm{ml})$, dried $\left(\mathrm{anh} . \mathrm{Na}_{2} \mathrm{SO}_{4}\right)$ and evaporated to afford the desired compound.

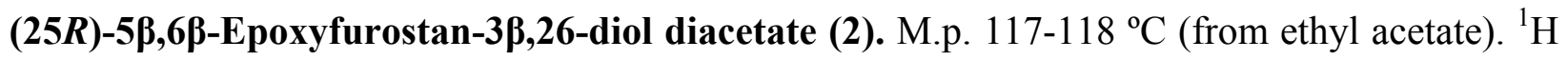
NMR $\left(400 \mathrm{MHz}, C D C l_{3}\right) \delta \mathrm{ppm} 4.82-4.67(\mathrm{~m}, 1 \mathrm{H}), 4.26(\mathrm{dt}, J=7.72,7.72,5.35 \mathrm{~Hz}, 1 \mathrm{H}), 3.89$ (ddd, $J=17.59,10.73,6.35 \mathrm{~Hz}, 1 \mathrm{H}), 3.27$ (dt, $J=8.20,8.16,4.05 \mathrm{~Hz}, 1 \mathrm{H}), 3.06$ (d, $J=2.10 \mathrm{~Hz}$, $1 \mathrm{H}), 2.03(\mathrm{~s}, 3 \mathrm{H}), 2.01(\mathrm{~s}, 3 \mathrm{H}), 1.00(\mathrm{~s}, 3 \mathrm{H}), 0.96(\mathrm{~d}, J=6.72 \mathrm{~Hz}, 3 \mathrm{H}), 0.91(\mathrm{~d}, J=6.73 \mathrm{~Hz}, 3 \mathrm{H})$, 0.74 (s, 3H). ${ }^{13} \mathrm{C}$ NMR (100 MHz) $\delta$ ppm $32.50 \mathrm{C}-1,27.14 \mathrm{C}-2,71.21 \mathrm{C}-3,37.92 \mathrm{C}-4,62.40 \mathrm{C}-$ 5, 63.34 C-6, 36.63 C-7, 29.37 C-8, 51.00 C-9, 35.12 C-10, 21.48 C-11, 39.42 C-12, 40.60 C-13, 56.30 C-14, 32.05 C-15, 83.00 C-16, 65.08 C-17, 16.26 C-18, 17.03 C-19, 37.87 C-20, 18.84 C21, 90.12 C-22, 30.41 C-23, 30.74 C-24, 32.73 C-25, 69.33 C-26, 16.72 C-27, 21.26 and 20.93 $\mathrm{CH}_{3} \mathrm{COO}, 171.23$ and $170.48 \mathrm{CH}_{3} \underline{\mathrm{COO}}$. MS (FAB): $516 \mathrm{M}^{+}, 515 \mathrm{M}^{+}-1,455,437,411,211,154$, 109, 95, 83, 81, 71, 69, 57, 55. HRMS (FAB): estimated for $\mathrm{C}_{31} \mathrm{H}_{47} \mathrm{O}_{6}\left(\mathrm{M}^{+}-\mathrm{H}\right)$ 515.3367, found 515.3364 .

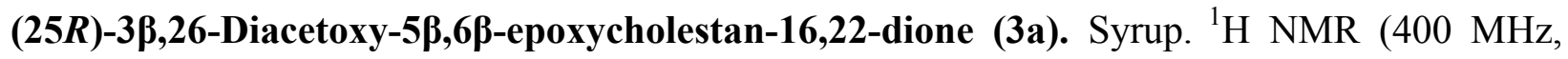
$\left.C D C_{3}\right) \delta$ ppm $4.74(\mathrm{tdd}, J=11.55,9.87,4.74,4.74 \mathrm{~Hz}, 1 \mathrm{H}), 4.00-3.81(\mathrm{~m}, 2 \mathrm{H}), 3.09(\mathrm{~d}, J=$ $2.31 \mathrm{~Hz}, 1 \mathrm{H}), 2.75(\mathrm{ddd}, J=17.83,9.47,5.59 \mathrm{~Hz}, 1 \mathrm{H}), 2.56$ (q, $J=5.88,5.86,5.86 \mathrm{~Hz}, 2 \mathrm{H})$, $2.03(\mathrm{~s}, 3 \mathrm{H}), 2.01(\mathrm{~s}, 3 \mathrm{H}), 1.00(\mathrm{~d}, J=7.55 \mathrm{~Hz}, 6 \mathrm{H}), 0.93(\mathrm{~d}, J=6.70 \mathrm{~Hz}, 3 \mathrm{H}), 0.73(\mathrm{~s}, 3 \mathrm{H}) .{ }^{13} \mathrm{C}$ NMR (100 MHz) $\delta$ ppm 32.39 C-1, 27.03 C-2, 71.05 C-3, 37.78 C-4, 62.45 C-5, 62.99 C-6, 36.41 C-7, 28.88 C-8, 50.61 C-9, 35.14 C-10, 21.26 C-11, 39.64 C-12, 41.53 C-13, 50.52 C-14, 38.55 C-15, 213.20 C-16, 66.11 C-17, 15.26 C-18, 16.95 C-19, 43.23 C-20, 12.81 C-21, 217.44 C-22, 37.05 C-23, 26.63 C-24, 32.00 C-25, 68.96 C-26, 16.72 C-27, 21.20 and $20.88 \mathrm{CH}_{3} \mathrm{COO}$, 171.20 and 170.41 $\mathrm{CH}_{3}$ COO. MS (FAB): $531 \mathrm{MH}^{+}, 515,513,472,471,411,295,229,223,211$, 115, 97, 107, 95, 91, 81, 69, 67, 55. HRMS (FAB) estimated for $\mathrm{C}_{31} \mathrm{H}_{47} \mathrm{O}_{7}\left(\mathrm{MH}^{+}\right)$531.3316, found 515.3272

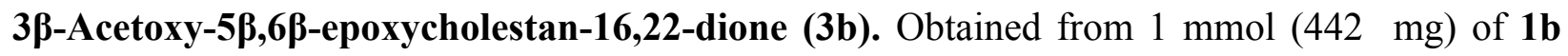
as described for $3 \mathbf{a}\left(\mathrm{KMnO}_{4} 5 \mathrm{~g}, \mathrm{Fe}_{2}\left(\mathrm{SO}_{4}\right)_{3} \cdot \mathrm{nH}_{2} \mathrm{O} 2.5, \mathrm{H}_{2} \mathrm{O} 0.5 \mathrm{ml}\right.$, reaction time $\left.120 \mathrm{~min}\right)$ yield $283.7 \mathrm{mg}$ (0.60 mmol, $60 \%)$ M.p. $134-135{ }^{\circ} \mathrm{C}$ (from $\left.\mathrm{MeOH}\right) .{ }^{1} \mathrm{H}$ NMR $\left(400 \mathrm{MHz}, \mathrm{CDCl}_{3}\right) \delta$ ppm: 4.74 (m,1H, H-3), 3.12 (a, H-6), 2.60 (d, $J=\mathrm{H}-17), 2.03$ (s, $\left.\underline{\mathrm{C}}_{3}-21\right), 0.99$ (s, $\left.\underline{\mathrm{C}}_{3}-19\right), 0.85$ (d, $\underline{\mathrm{CH}}_{3}-26$ y $\left.\underline{\mathrm{H}}_{3}-27\right), 0.74$ (s, $\left.\underline{\mathrm{CH}}_{3}-18\right) .{ }^{13} \mathrm{C}-\mathrm{NMR}(100 \mathrm{MHz}): 32.39 \mathrm{C}-1,27.04 \mathrm{C}-2,71.07 \mathrm{C}-3$, 37.79 C-4, 62.48 C-5, 63.04 C-6, 36.42 C-7, 28.88 C-8, 50.59 C-9, 35.14 C-10, 21.27 C-11, 40.40 C-12, 41.54 C-13, 50.53 C-14, 38.55 C-15, 213.89 C-16, 66.03 C-17, 15.31 C-18, 16.97 C19, 43.20 C-20, 12.82 C-21, 217.44 C-22, 37.09 C-23, 32.14 C-24, 27.57 C-25, 22.39 C-26, $22.39 \mathrm{C}-27,21.22 \mathrm{CH}_{3} \mathrm{COO}, 170.44 \mathrm{CH}_{3} \underline{\mathrm{COO}}$. MS (FAB): $473 \mathrm{MH}^{+}, 413,395,327,267,207$, 147, 83, 81, 69. HRMS (FAB) estimated for $\mathrm{C}_{29} \mathrm{H}_{45} \mathrm{O}_{5}\left(\mathrm{MH}^{+}\right)$473.3262, found 473.3303 .

(25R)-3ß,26-Diacetoxy-cholest-5-en-16,22-dione, krytogenin acetate (4b). A solution of 3a (400 mg, $0.73 \mathrm{mmol})$ in $\mathrm{CH}_{2} \mathrm{Cl}_{2}(20 \mathrm{ml})$ was shaken for $20 \mathrm{~min}$ with $48 \% \mathrm{HBr}(10 \mathrm{ml})$. The organic layer was washed with water $(5 \times 10 \mathrm{ml})$, dried $\left(\mathrm{anh} . \mathrm{Na}_{2} \mathrm{SO}_{4}\right)$ and evaporated. The 
residue was refluxed for $30 \mathrm{~min}$. with $\mathrm{Zn}$ dust $(950 \mathrm{mg})$ in $\mathrm{AcOH}(15 \mathrm{ml})$. Ethyl acetate was added, the resulting mixture was filtered and washed with $10 \%$ aqueous $\mathrm{NaCl}(5 \times 10 \mathrm{ml})$, water $(3 \times 10 \mathrm{ml}), 5 \%$ aqueous $\mathrm{Na}_{2} \mathrm{CO}_{3}\left(10 \mathrm{ml}\right.$ portions until evolution of $\mathrm{CO}_{2}$ ceased), $10 \%$ aqueous $\mathrm{NaCl}(2 \times 10 \mathrm{ml})$, dried (anh. $\left.\mathrm{Na}_{2} \mathrm{SO}_{4}\right)$ and evaporated to afford 5, (224.4 mg, $57.8 \%$ ) after chromatographic purification in silica gel. M.p. $143-144{ }^{\circ} \mathrm{C}$ (from ethyl acetate); lit. ${ }^{7} 152-153$ ${ }^{\circ} \mathrm{C} .{ }^{1} \mathrm{H}$ NMR (400 MHz, CDCl $)$ ), $\delta$ ppm 5.36 (d, $\left.J=4.83 \mathrm{~Hz}, 1 \mathrm{H}, \mathrm{H}-6\right), 4.67-4.52$ (m, 1H, H-3), $3.93(\mathrm{dd}, J=6.07,3.08 \mathrm{~Hz}, 2 \mathrm{H}, \mathrm{H}-26), 2.77$ (m, 1H, H-20), 2.04 (s, 3H, $\mathrm{CH}_{3}$ acetyl), 2.02 (s, $3 \mathrm{H}, \mathrm{CH}_{3}$ acetyl), 1.04 (m, 6H, H-19 and H-21), 0.95 (d, $\left.J=6.72 \mathrm{~Hz}, 3 \mathrm{H}, \mathrm{H}-21\right), 0.79$ (s, 3H, H18). ${ }^{13} \mathrm{C}$ NMR $(100 \mathrm{MHz}) \delta$ ppm $36.61 \mathrm{C}-1,27.59 \mathrm{C}-2,73.64 \mathrm{C}-3,37.97 \mathrm{C}-4,139.83 \mathrm{C}-5$, 121.73 C-6, 31.66 C-7, 30.87 C-8, 49.53 C-9, 36.61 C-10, 20.44 C-11, 39.61 C-12, 41.61 C-13, 51.10 C-14, 38.51 C-15, 213.20 C-16, 66.09 C-17, 15.33 C-18, 19.25 C-19, 43.31 C-20, 12.92 C21, 217.86 C-22, 37.13 C-23, 26.65 C-24, 32.02 C-25, 68.97 C-26, 16.76 C-27, 21.33 and 20.90 $\mathrm{CH}_{3} \mathrm{COO}, 171.19$ and $170.40 \mathrm{CH}_{3} \underline{\mathrm{COO}}$.

3及-Acetoxy-cholest-5-en-16,22-dione (4b). Obtained in $56.5 \%$ (117 $\mathrm{mg}, 0.26 \mathrm{mmol})$ from $\mathbf{3 b}$ (215.9 mg, $0.46 \mathrm{mmol}$ ) as described for kryptogenin acetate (4a). M.p. $152-154{ }^{\circ} \mathrm{C}$ (from $\mathrm{MeOH}$ ) lit. ${ }^{4 \mathrm{a}} 151-152{ }^{\circ} \mathrm{C} .{ }^{1} \mathrm{H}$ NMR $\left(400 \mathrm{MHz}, \mathrm{CDCl}_{3}\right) \delta \mathrm{ppm}$ : $5.36(\mathrm{~d}, J=4.8 \mathrm{~Hz}, \mathrm{H}-6), 4.65-4.54(\mathrm{~m}, \mathrm{H}-$ 3), 2.61 (d, $J=3.6 \mathrm{~Hz}, \mathrm{H}-17), 2.02$ (s, $\underline{\mathrm{H}}_{3} \mathrm{COO}-3$ ), 1.03 (s, $\left.\underline{\mathrm{C}}_{3}-19\right), 0.89$ (d, J=6 Hz, $\underline{\mathrm{C}}_{3}-26$ y $\left.\mathrm{C}_{3}-27\right), 0.79\left(\mathrm{~s}, \mathrm{CH}_{3}-18\right){ }^{13} \mathrm{C}$ NMR $(100 \mathrm{MHz}): 31.67 \mathrm{C}-1,27.60 \mathrm{C}-2,73.66 \mathrm{C}-3,37.98 \mathrm{C}-4$, 139.81 C-5, 121.76 C-6, 36.62 C-7, 30.88 C-8, 51.09 C-9, 36.62 C-10, 20.45 C-11, 38.51 C-12, 41.62 C-13, 49.54 C-14, 40.38 C-15, 217.85 C-16, 66.01 C-17, 12.94 C-18, 19.26 C-19, 43.29 C20, 15.37 C-21, 213.86 C-22, 37.16 C-23, 32.17 C-24, 27.60 C-25, 21.36 C-26, 22.41 C-27, $22.41 \underline{\mathrm{CH}}_{3} \mathrm{COO}, 170.42 \mathrm{CH}_{3} \underline{\mathrm{COO}}$.

\section{Acknowledgements}

We thank Dirección General de Asuntos del Personal Académico (DGAPA-UNAM) for financial support via project IN-204008 and Dirección General de Estudios de Postgrado (DGEP-UNAM) for the scholarship granted to A.R-A. We are indebted to Rosa I. del Villar Morales and Margarita Guzmán Villanueva (USAI-UNAM) for registering NMR and Mass spectra.

\section{References}

1. (a) Syamala, M. S.; Das, J.; Baskaran, S.; Chandrasekaran, S. J. Org. Chem. 1992, 57, 19281930. (b) Parish, E. J.; Li, H.; Li, S. Synth. Commun. 1995, 25, 927. (c) Hanson, J. R.; Hitchock, P. B.; Liman, M. D; Nagaratnam, S.; Manickavasagar, R. J. Chem. Res. (S) 1995, 220. (d) Parish, E. J.; Li, S. J. Chem. Res. (S) 1996, 288. (e) Parish, E. J.; Li, S. J. Org. Chem. 1996, 61, 5665. (f) Salvador, J. A. R.; Sáe Melo, M. L.; Campos Neves, A. S. 
Tetrahedron Lett. 1996, 37, 687. (g) Salvador, J. A. R.; Hanson, J. R. J. Chem. Res. (S) 2002, 576. (h) Silvestre, S. M.; Salvador, J. A. R.; Clark, J. H. J. Mol. Catal. A: Chemical 2004, 219, 143.

2. Iglesias-Arteaga, M. A.; Símuta-Lopez, E. M.; Xochihua-Moreno, S.; Viñas-Bravo, O.; Montiel-Smith, S.; Meza Reyes, S.; Sandoval-Ramírez, J. J. Braz. Chem. Soc. 2005, 16(3A), 381.

3. (a) González, A. G.; Francisco, C. G.; Freire, R.; Hernández, R.; Salazar, J. A.; Suárez, E. Tetrahedron Lett. 1974, 15, 4289. (b) Iglesias-Arteaga, M. A.; Mendez-Stivalet, J. M.; Pérez, N. Nat. Prod. Commun. 2007, 2(1), 47.

4. (a) Chausuancharoen, N.; Kongkathip, N.; Konkathip, B. Synth. Commun. 2004, 34, 961983. (b) Xu, Q.; Peng, X-w.; Tian, W-s. Tetrahedron Lett. 2003, 44, 9375.

5. Kryptogenin and its derivatives have been employed as starting material for the synthesis of different bioactive steroid, see (a) Mui, M. M.; Kamat, S. Y.; Elliott, W. H. Steroids, 1974, 24, 239. (b) Cheng, M.S.; Wang, Q. L.; Tian, Q.; Song, H.Y.; Liu, Y.X.; Li, Q.; Xu, X.; Miao, H. D.; Yao, X. S.; Yang, Z. J. Org. Chem. 2003, 68, 3658. (c) Laura Alessandrini, L.; Ciuffreda, P.; Santaniello, E.; Terraneo, G. Steroids, 2004, 69, 789. (d) Liu, Y.; Zhao, D-M.; Lu, X-H.; Wang, H.; Chen, H.; Ke, Y.; Leng, L.; Cheng, M-S. Bioorg. Med Chem. Lett 2007, 17(11), 156.

6. Furostanic compounds can be obtained from steroid sapogenins by different reductive treatments a) $\mathrm{LiAlH}_{4} / \mathrm{AlCl}_{3}$ in diethyl ether Iglesias-Arteaga, M. A.; Pérez-Gil, R.; LeliebreLara, V.; Pérez-Martínez, C. S.; Coll, F. J. Chem. Res. (S) 1996, 504. (b) hydrogenation over $\mathrm{PtO}_{2}$ in acidic media Iglesias-Arteaga, M. A.; Perez-Gil R.; Leliebre-Lara, V.; PerezMartínez C. S.; Coll-Manchado, F. Synth. Commun. 1998, 28, 1779. (c) $\mathrm{NaBH}_{3} \mathrm{CN}$ reduction in acetic acid Chausuancharoen N, Kongkathip N, Konkathip B. Synth. Commun. 2004, 34, 961. (d) Romero-Avila, M.; de Dios-Bravo, M. G.; Mendez-Stivalet, J. M.; RodríguezSotres, R.; Iglesias-Arteaga, M. A. Steroids 2007, 72, 955.

7. Marker, R. E.; Wahner, R.B.; Ulshafer, P. R.; Wittbecker, E. L.; Goldsmith, D. P. J; Raouf, C. H. J. Am. Chem Soc. 1947, 69, 2167. 